\title{
Relationship between PET metabolism and SEEG epileptogenicity in focal
}

lesional epilepsy

\section{Authors:}

Stanislas LAGARDE ${ }^{1}$, Mohamed BOUCEKINE ${ }^{2}$, Aileen MCGONIGAL ${ }^{1}$, Romain CARRON $^{3}$, Didier SCAVARDA ${ }^{4}$, Agnès TREBUCHON ${ }^{1}$, Mathieu MILH $^{5}$, Laurent BOYER ${ }^{2}$, Fabrice BARTOLOMEI ${ }^{1}$, Eric GUEDJ $6,7,8$

Mis en forme : Justifié

\section{Affiliations:}

1. Aix Marseille Univ, APHM, INSERM, INS, Inst Neurosci Syst, Timone Hospital, Epileptology Department, Marseille, France;

2. Aix Marseille Univ, EA 3279 CEReSS - Health Service Research and Quality of Life Center, Marseille, France

3. Aix Marseille Univ, APHM, INSERM, INS, Inst Neurosci Syst, Timone Hospital, Functional and Stereotactic Neurosurgery, Marseille, France;

4. APHM, Timone Hospital, Pediatric Neurosurgery, Marseille, France;

5. APHM, Timone Hospital, Pediatric Neurology, Marseille, France;

6. APHM, Timone Hospital, Nuclear Medicine Department, Marseille, France

7. Aix Marseille Univ, CNRS, Ecole Centrale Marseille, UMR 7249, Institut Fresnel, Marseille, France

8. Aix Marseille Univ, CERIMED, Marseille, France Corresponding Author: Eric Guedj, MD, PhD

eric.guedj@ap-hm.fr ; 
Service Central de Biophysique et Médecine Nucléaire, Hôpital de la Timone, 264 rue Saint Pierre, 13005 Marseille, France.

Tel.: +33-491385558; Fax: +33-491384769

\section{ABSTRACT}

Purpose: This study aims to evaluate the performance of ${ }^{18} \mathrm{~F}$-FDG PET for distinguishing the epileptogenic zone (EZ) from propagation and non-involved zones at brain area level, as defined using stereo-EEG, in patients with pharmacoresistant epilepsy due to malformations of cortical development (MCD). Additionally, we seek to determine the relationship between ${ }^{18} \mathrm{~F}-\mathrm{FDG}$-PET data and post-surgical seizure outcome.

Methods: Thirty-eight patients with MCD were explored with ${ }^{18} \mathrm{~F}$-FDG PET and stereo-EEG. We compared PET metabolism of each patient to a control-population of healthy subjects. Based on MRI and SEEG, we separated 4 distinct zones at individual level: lesional, epileptogenic non-lesional, propagation, and non-involved. Then, we analysed: 1) difference of PET metabolism within these four distinct zones; 2) performance of PET in defining the EZ within the SEEG-sampled areas; 3) relation between extension of PET hypometabolism and post-surgical seizure outcome.

Results: We found: 1) a gradient of PET hypometabolism from non-involved to propagation, then to epileptogenic and lesional zones $(p<0.001) ; 2$ ) good performance of PET in defining the EZ ( $A U C$ of ROC curve $=0.82$ ); 3) poorer post-surgical prognosis associated with PET hypometabolism extension beyond SEEG sampling $(p=0.024)$.

Conclusion: ${ }^{18}$ F-FDG-PET has good accuracy in determining EZ in patients with MCD even if the hypometabolism is not limited to the EZ. Furthermore, hypometabolic extension is unfavourably associated with post-surgical prognosis. 


\section{MANUSCRIPT}

\section{INTRODUCTION}

Malformations of cortical development (MCDs), including focal cortical dysplasia (FCD) and neurodevelopmental tumour (NDT), are frequent causes of pharmacoresistant epilepsy. MCD are the third most frequent aetiology in epilepsy surgical cases[1], representing about $20 \%$ of all operated patients, with increasing prevalence over time[2,3]. These patients are often good candidates for epilepsy surgery with favourable seizure outcome[2]: about $65 \%$ of patients are Engel Class I in case of FCD[4,5], and up to $80 \%$ in case of NDTs[6,7].

The International League Against Epilepsy (ILAE) classification[8] divides FCD into three subtypes (type I, II, and III) characterized by cortical architectural abnormalities, presence or not of dysmorphic ectopic neurons, and presence or not of an associated lesion (e.g. hippocampal sclerosis). NDTs are the second most prevalent MCD, essentially represented by dysembryoplastic neuroepithelial tumours (DNET) and gangliogliomas[9]. They are classified in the World Health Association (WHO) Classification of the Central Nervous System as grade I tumours of neuroepithelial tissue, neuronal, and mixed neuronal-glial tumorstumours. [10] NDT are often associated with cytoarchitectural changes within adjacent cortex, with similarities to FCD, and these cases are grouped as FCD type IIIb in ILAE classification.

Many patients with epilepsy related to MCD can be operated based on video-EEG and multimodal imaging data (magnetic resonance imaging (MRI), positron emission tomography (PET))[4], without invasive exploration. Nevertheless, stereo-electroencephalography (stereoEEG, SEEG) remains mandatory in some clinical situations: negative or non-contributory MRI, 
discordance between lesion and hypothesis about the epileptogenic zone (EZ), or proximity to eloquent areas[11].

In this context, interictal ${ }^{18} \mathrm{~F}$-fluorodeoxyglucose-PET $\left({ }^{18} \mathrm{~F}\right.$-FDG-PET) is of particular importance in helping to localize and delineate MCD, especially in cases of negative or doubtful MRI, with the use of PET/MRI co-registration [12-15]. Across series, ${ }^{18}$ F-FDG PET detects areas of hypometabolism in $60-92 \%$ of epilepsy cases with FCD [4,16-19]. However, ${ }^{18}$ F-FDG PET hypometabolism is frequently more extensive than the MRI lesion[20], showing regional rather than focal hypometabolism[13,14,21,22]. Relation between epileptogenicity and PET hypometabolism may thus remain unclear in such patients[22-24].

Furthermore, the EZ is not confined to the lesional area in some MCD cases [25]. This is particularly true for FCD type I, but also for a significant number of FCD II and NDT, which can have a network organization of EZ. For example, 33/74 patients (44\%) with MCD operated in our centre had a complex organization of the EZ including more than one epileptogenic area[26].

In order to investigate this issue, it is crucial to precisely correlate PET findings with accurate definition of regions involved in seizure onset and propagation. However, previous studies have been mostly performed at patient-level and not at brain area-level. Here, we report on a series of patients with MCD explored with SEEG, studying the relationships between epileptogenicity and ${ }^{18}$ F-FDG PET data. Our aim was to evaluate the performance of ${ }^{18} \mathrm{~F}$-FDG PET in distinguishing EZ as defined using SEEG criteria, and its determinants. We also wished to investigate whether ${ }^{18} \mathrm{~F}$-FDG PET data was correlated with SEEG-defined propagation zone. Additionally, we seek to determine the relationship between ${ }^{18}$ F-FDG-PET data and postsurgical seizure outcome. 


\section{METHODS}

\section{Subjects}

Among all patients who underwent stereotactic-EEG (SEEG) in our department from $200 \underline{4} \theta$ to 20146, and explored by a same PET camera (see below), we reviewed all patients with pathologically proven focal cortical dysplasia (FCD) or neurodevelopmental tumours (NDT).

An expert neuropathologist reviewed data and established the pathological diagnosis according to International League Against Epilepsy (ILAE) [8] and WHO classifications[10]. We included patients with isolated FCD type I or type II, and patients with neurodevelopmental tumours (NDT), with a total of 38 patients analysed. The institutional review board of the French Institute of Health (IRB15226) approved this study and written patient consent was obtained.

All patients had detailed evaluation including medical history, neurological examination, cognitive testing, cerebral ${ }^{18} \mathrm{~F}-\mathrm{FDG}-\mathrm{PET}$ and MRI. All patients required invasive SEEG exploration after the non-invasive phase as part of patients' usual clinical care. StereotacticEEG was indicated when the EZ was suspected to be larger than the lesion; and/or when extratemporal structures were suspected to be involved in possible temporal lobe epilepsy; and/or when bilateral organization was suspected; and/or when a functional area was suspected to be involved; and/or when no visible MRI lesion existed[27].

Surgical outcome was regularly assessed during postoperative follow-up according to Engel's classification [28]. For analysis, we used surgical outcome score at last available follow-up and defined two groups: favourable (Engel I + II) and unfavourable (Engel III + IV) outcome.

Finally, a group of 34 healthy subjects ( $38.2 \pm 9.8$ years, 20 women) was extracted from a local normal ${ }^{18} \mathrm{~F}$-FDG-PET database constituting a control population (Clinical Trials Ref: 
NCT00484523) approved by ethics committee. These controls were free of neurological and psychiatric disease, and had normal brain MRI.

\section{SEEG recordings}

Placement of electrodes was defined in each patient based on non-invasive information providing hypotheses about the potential localization of the EZ. Long-term video-SEEG monitoring was performed to record several of the patient's usual seizures, following complete or partial withdrawal of antiepileptic drugs. The SEEG exploration was performed using intracerebral multiple-contact electrodes (Dixi Medical or Alcis), consisting of 10-15 contacts with length $2 \mathrm{~mm}$, diameter $0.8 \mathrm{~mm}$, spaced by $1.5 \mathrm{~mm}$ (for details see [29]).

We first selected a subset of all stereotactic-EEG channels. To this purpose, we co-registered pre-implantation MRI and post-implantation $\mathrm{CT}$ (using the maximization of normalized mutual information and trilinear interpolation) [30], and visually defined the anatomical area using the 116 regions of the AAL atlas[31]. First, we selected only the contacts within the grey matter, because the exact origin of the signal recorded in white matter is not well understood. Second, to limit the influence of not completely inactive common reference and biases due to volume conduction effects, we choose a bipolar re-referencing (obtained from subtraction of monopolar signals recorded on two adjacent contacts in the structure) rather than monopolar signals. Third, in cases of more than two channels within the same brain area, we selected the channel without artefact and with the higher epileptogenicity index (EI)[29].

Based on ictal activity, we labelled SEEG contacts as belonging to Epileptogenic Zone (EZ), Propagation Zone (PZ) or Non-Involved Zone (NIZ) according to previous studies[29,32]. We defined the EZ using visual analysis complemented by a quantitative method, the 
Epileptogenicity Index (EI). This index is a semi-automatic method to quantify the dynamic of the fast-activity genesis at the beginning of seizure. It is based on two important features of the transition from pre-ictal to ictal activity: (i) redistribution of signal energy from lower frequency bands (theta, alpha) toward higher frequency bands (beta, gamma); and (ii) delay of appearance of these high frequencies. The sooner a structure becomes involved in the seizure, and the faster its ictal discharge, the higher its El. To obtain normalized values per patient, ranging from 0 (no epileptogenicity) to 1 (maximal epileptogenicity), El values were divided by the maximal value obtained in each patient (for more details regarding methodology see [33]). We defined the EZ as all brain areas with El $\geq 0.3$. This cut-off was chosen pragmatically because it allows the best separation between epileptogenic and nonepileptogenic areas, as demonstrated in previous reports [33]. In cases of low frequency seizure onset pattern (below $12 \mathrm{~Hz}$ ), the EZ was defined visually as the structures involved within the first 5 seconds.

Propagation zone (PZ) was defined by visual analysis as brain areas with an $\mathrm{El}<0.3$ but with sustained discharge during the course of the seizure (including rhythmic discharges with propagation delay and/or low frequency)[34]. In cases of low frequency seizure onset pattern (below $12 \mathrm{~Hz}$ ), the PZ was defined visually as the structures involved after the first 5 seconds. The non-involved zone (NIZ) was defined as all other brain structures sampled by SEEG. The lesional area(s) was defined based on the MRI (only for the patients with visible MRI lesion). All these zones were defined at the level of Automated Anatomical Labelling (AAL) atlas regions[31].

Extension of the EZ was evaluated as the number of distinct brain regions (further referred to as "sub-lobes") involved. We distinguished the following sub-lobes: mesial temporal, lateral temporal, insular, orbitofrontal, mesial prefrontal, lateral prefrontal, premotor, central (pre- 
and postcentral), opercula (frontal and rolandic), mesial parietal, lateral parietal, mesial occipital, and lateral occipital. Focal organization corresponded to a situation where EZ remained confined only to one of the sub-lobes defined above; in other cases, the EZ was considered to be network-organized.

\section{${ }^{18}$ F-FDG PET acquisition}

${ }^{18}$ F-FDG PET scans were performed under the same conditions for all subjects, including healthy controls. An integrated PET/CT camera (Discovery ST, GE Healthcare, Waukesha, WI) was used with an axial resolution of $6.2 \mathrm{~mm}$ allowing 47 contiguous transverse sections of the brain of $3.27 \mathrm{~mm}$ thickness. ${ }^{18} \mathrm{~F}-\mathrm{FDG}(150 \mathrm{MBq})$ was injected intravenously, in an awake and resting state with eyes closed in a quiet environment, in an interictal period for patients. Image acquisition started 30 min after injection and ended 15 min thereafter. Images were reconstructed using the ordered subsets expectation maximization algorithm with 5 iterations and 32 subsets and corrected for attenuation using a CT transmission scan.

\section{${ }^{18}$ F-FDG PET analysis}

Brain statistical analysis was performed at voxel-level using SPM8 software (Wellcome Department of Cognitive Neurology, University College, London, UK) to compare each individual patient to the group of 34 healthy subjects considering age and gender as covariables. The PET images were spatially normalized onto the Montreal Neurological Institute $(\mathrm{MNI})$ atlas. The dimensions of the resulting voxels were $2 \times 2 \times 2 \mathrm{~mm}$. The images were then smoothed with a Gaussian filter (8mm full-width at half-maximum) to blur individual variations in gyral anatomy and to increase the signal-to-noise ratio. Two options for intensity normalization were evaluated to correct individual variations in global brain metabolism: 1 / 
the proportional scaling, giving the same global metabolic value to each PET examination; 2/ the cerebellar scaling, giving the same cerebellar metabolic value to each PET examination. The analysis performed in comparison to healthy subjects was thereafter individually conducted patient by patient at two levels: 1 / at region-of-interest (ROI)-level,, limiting the analysis to the location of SEEG recordings according to the AAL atlas, 2/ at whole-brain level, without considering the specific location of SEEG electrodes.

\section{$\underline{\text { ROI PET analysis }}$}

The following parameters were extracted from SPM(T) maps at individual level of each patient within the AAL ROI corresponding to SEEG sampling: average metabolic value, asymmetric metabolic index ([average left region - average right region]/[average left region + average right region], and hypometabolic voxel T-peak. The hypometabolic voxel T-peak corresponds to the T-score of the most significant voxel of the evaluated region when comparing the individual subject to the group of healthy controls. Concerning this latter, a normalized parameter was also evaluated given the value of 1 to the most metabolically affected electrode in each patient, similarly to the normalization done for the epileptogenicity index (i.e. the "hypometabolic index").

\section{Whole-brain PET analysis}

\section{The SPM(T) maps were obtained by comparing each individual patient to the group of healthy}

controls, The between-groups SPM(T) maps were obtained-at a height threshold (voxel-level significance) of $p<0.005$, corrected for the cluster volume after Monte-Carlo simulations $(k>119)$ to avoid type II errors as recommended[35]. Xjview (http://www.alivelearn.net/xjview/) and AAL atlas were used to determine brain 
hypometabolic regions involved, and specifically their extension (number of regions with regards to the $116 \mathrm{AAL}$ regions).

\section{Statistical Methods}

We performed 2 sets of statistical analyses, asking 4 distinct questions:

1. At the ROI level, within the SEEG-sampled areas: (1) What is the performance of PET in differentiating epileptogenic from other zones? (2) What is/are the determinant(s) of the PET hypometabolism?

2. At the whole-brain level: (1) What is/are the determinant(s) of the PET hypometabolism spatial extension? (2) Are any PET features associated with postsurgical seizure prognosis?

For the analysis of PET performance, we first performed univariate testing, and in cases of multiple significant associations/correlations we secondly used multivariate analyses. For the classification as EZ or NIZ based on PET, a stepwise logistic regression with forward elimination was performed on the overall sample to identify predictors of high epileptogenicity of structures. The original dataset was randomly split into training $(2 / 3)$ and test datasets $(1 / 3)$. The training set is used for model development, and the test set is used to assess the model accuracy. Multiple logistic regression models were built. Each variable was examined by univariate analysis and included in the model when the $P$ value was $<0.20$. Subsequently, multivariate logistic regression with forward elimination of any variable that did not contribute to the model was performed. Odds ratios (OR) and $95 \%$ confidence intervals $(95 \% \mathrm{Cl})$ are reported. A two-sided $p$-value of $<0.05$ was considered to indicate statistical significance. The sensitivity, specificity and area under the curve (AUC) were calculated, using 
receiver operating characteristic (ROC) for the final model. Based on a rough classifying system, AUC can be interpreted as follows: $90-100=$ excellent; $80-90=$ good; $70-80=$ fair; $60-70=$ poor $; 50-60=$ fail

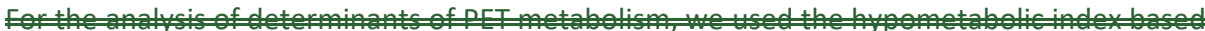

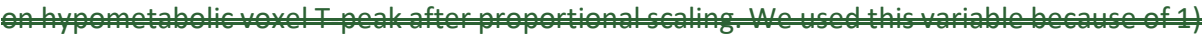

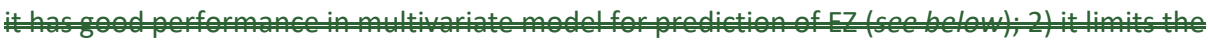

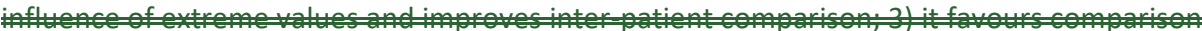
7. For analyses of determinants of PET hypometabolism intensity, PET hypometabolism extension and relation with post-surgical outcome, we firstly perform appropriate univariate analysis and secondly build a multivariate analysis (ANOVA).

In order to evaluate the variation in the SEEG spatial sampling according to the patients features, we tested for statistical significance the number of sampled regions against the following factors: histological type, MRI findings, age at epilepsy onset, age at SEEG, epilepsy duration and post-surgical seizure outcome._Categorical variables are presented as numbers and percentages, and continuous variables are presented as means \pm SD. For the categorical variables the significance of differences was determined by the chi-square test or Fisher's exact test, and by the t-test or Mann-Whitney or Kruskal-Wallis test for continuous variable, according to normality assumption.

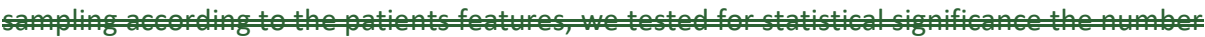

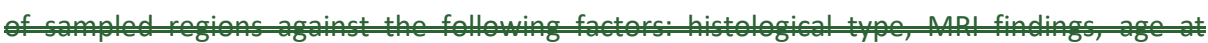
epilepsy onset, age at SEEG, epilepsy duation and post surgical seizure outcome, The statistical analyses were performed using IBM Corp Released 2012. IBM SPSS Statistics for Windows, Version 21.0. Armonk, NY: IBM Corp. 


\section{RESULTS}

\section{Patient features}

Table 1 summarizes population features.

A total of 443 AAL regions sampled by SEEG electrodes were analysed (mean per patient $=$ $11.7 \pm 3.1)$. There was no significant association between the number of sampled regions and the following factors: histological types, MRI findings, age at epilepsy onset, age at SEEG, and post-surgical seizure outcome. In our series, patients with longer epilepsy duration had more widespread SEEG sampling ( $R=0.33, p=0.031$, Spearman correlation).

One hundred and forty-four AAL regions belonged to the epileptogenic zone (including 33 within the MCD), 107 to the propagation zone, and 212 to the non-involved zone. Mean number of areas within the EZ was $3.3 \pm 2$ and within the PZ was $2.8 \pm 2$. There was no significant association between the number of areas belonging to the EZ and the following factors: histological types, MRI findings, age at epilepsy onset, epilepsy duration, age at SEEG, and post-surgical seizure outcome.

\section{SEEG / PET correlation}

Within the areas sampled by SEEG, the following variables were significantly associated with the classification between EZ and other zones (PZ+NIZ) after univariate testing: PET hypometabolism (all normalization methods except one, the metabolic index with cerebellar scaling, also including the evaluation of asymmetry), EZ localization, histological type (Table 2). A multivariate model-analysis was then built-perform including these variables with a pfrom univariate statistical tests (adding to the previous significant variables the two following: gender and MRI-findings). After this multivariate analysis, only the 2 following factors 
remained significantly associated with the classification as EZ or NIZ based on SEEG: hypometabolic voxel T-peak normalized cerebellar activity, and hypometabolic index normalized on global activity. The final statistical model included then these two variables and had the following formula:

$$
P(E Z=\text { yes })=\frac{e^{-3.359+1.587 \times \text { Hypometabolic Index }+0.387 \times \text { T-Peak }}}{1+e^{-3.359+1.587 \times \text { Hypometabolic Index }+0.387 \times T-\text { Peak }}}
$$

where: Hypometabolic Index is the hypometabolic index normalized on global activity and $T$ Peak is the hypometabolic voxel T-peak normalized on cerebellar activity. Optimal cut-off probability to predict the class of ROI was determined by the Youden index which is the difference between true positive rate and false positive rate over all possible cut-point values $[36,37]$. The optimal cut-off of our model was 0.3296 . Thus, if one ROI have a probability above

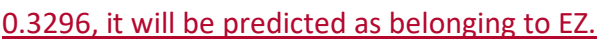

Overall, on the test sample, automatic classification of epileptogenic versus non-epileptogenic regions based on PET imaging performed well with for the forward selection with an AUC of 0.82 (0.74-0.90); with a sensitivity of 65.8 (50.5-78.4); specificity of 67.8 (80.3-92.8); PPV of 67.5 (52-79.9); and NPV of 87 (79.4-92.1) (Figure 1). The figure 2 illustrates patient by patient and ROI by ROI the correspondence between model prediction (based on PET) and SEEG epileptogenicity.

Concerning the misclassified areas, within the areas considered as epileptogenic based on PET but not on SEEG data (False Positive): $50 \%$ of them belonged to the propagation zone and $50 \%$ to the NIZ. Taken as a whole, $22.4 \%$ of all areas of the propagation zone were classified as epileptogenic based on PET data.

\section{Determinants of PET hypometabolism}


Determinants of PET hypometabolism were sought based on the two PET variables previously identified for the classification (i.e. the hypometabolic voxel T-peak normalized on cerebellar activity, and the hypometabolic index normalized on global activity-). For the analysis of Mis en forme : Couleur de police : Rouge determinants of PET metabolism, we used the hypometabolic index based on hypometabolic voxel T-peak after proportional scaling. used this it has performance in multivariate model for prediction of $E Z$ (see below); 2 ) it limits the influence of extreme values and improves inter-patient comparison; 3) it favours comparison with the Epileptogenicity Index used in clinical practice (since El is also normalized between 0 and 1). Within the areas sampled by SEEG, the following factors were associated on univariate tests with the hypometabolic index: histological type ( $p=0.02$, Kruskal-Wallis), MRI findings (presence or not of a visible MCD, $p=0.005$, Mann-Whitney), and category of the zone ( $p<0.001$, Kruskal-Wallis). On multivariate analysis, all three determinants remained significantly associated with the hypometabolic index: 1$)$ category of the zone (F=20.4, $p<0.001), 2)$ histological type $(F=5.5, p=0.04), 3)$ MRI findings (visible lesion or not) $(F=5.4$, $p=0.021$ ). Concerning the MRI findings, the MRI-positive cases had lower global hypometabolic index within all SEEG sampled areas.Concerning the zone studied, we found a gradual increase of hypometabolic index from the NIZ, to PZ, to EZ, to Lesion. This means that lesion is more hypometabolic than flesion $\left.>E Z \_-p<0.001\right)$; EZ is more hypometabolic than $\left.>P Z \_-p<0.001\right) ; P Z$ is more hypometabolic than $\left.>N I Z \_\div-p=0.001\right) ; E Z$ is more hypometabolic than $>N I Z_{-} \div(p<0.001)$; Itesion is more hypometabolic than $\left.>P Z \_--p<0.001\right)$; and Itesion is more hypometabolic than $>\mathrm{NIZ}(\div \mathrm{p}>0.001$, Figure $3 \mathrm{~A})$. This pattern was, with similar pattern acrossfor all histological types _(no significant interaction on multivariate analysis, Figure 3B). Concerning the MRI findings, the MRI-positive cases had higher metabolism (lower hypometabolic index) of PZ and NIZ than MRI-negative cases ( $p=0.009$ and $p<0.001$ 


\section{Hypometabolism extension and prognostic value of PET}

On the whole-brain PET analysis, the mean number of significantly hypometabolic cortical regions on PET $(p<0.005, k>119)$ was 20.1 ( \pm 14.7 ) per patient (defined on AAL atlas), being clearly larger than the number of epileptogenic regions (mean $=3.3$ ). In order to control for variability in SEEG sampling between patients, we performed statistical testing using the percentage of significantly hypometabolic areas within all areas not sampled by SEEG. There was no significant association with the following clinical variables: histological type, MRI findings, type of organization of EZ (network or focal).

In this series, there was no significant association between outcome and the following clinical factors: histological type, visibility of MRI visible lesion, epilepsy duration, and the intensity of PET hypometabolism. Nevertheless, we found that the extension of PET hypometabolism outside SEEG sampling was associated with poorer post-surgical seizure outcome. Precisely, the percentage of significantly hypometabolic zone among all not sampled AAL areas was significantly higher in the patients with unfavourable seizure outcome after surgery (Engel III+IV, $p=0.02$, Kruskal-Wallis test, Figures 4-5). Notably, the results were similar comparing patients being Engel I or Engel II-III-IV after surgery.

\section{DISCUSSION}


In this study, we compared SEEG and ${ }^{18}$ FDG-PET data in patients with focal epilepsy associated with MCD. We highlight several points: 1) PET has good performance in defining the epileptogenic zone (AUC=0.82); 2) there is a PET hypometabolism gradient, with increasing hypometabolism from non-involved to propagation zones, then to epileptogenic and lesional zones; 3 ) the gradient of PET hypometabolic index remains similar across histological type but is more marked in MRI-positive cases; 4) the extension of PET hypometabolic area beyond the limits of SEEG exploration is unfavourably associated with post-surgical prognosis.

\section{Value of PET in determining EZ in MCDs}

Previous studies have demonstrated that ${ }^{18}$ FDG-PET detects areas of hypometabolism localizing the FCD/epileptic areas in $60-92 \%$ of cases $[4,16-19]$. Moreover, PET/MRI coregistration allow detection of $95-98 \%$ of FCD in operated patients[12,38][12,38], precluding the estimation of the prevalence of false-negative cases. However, until now, previous studies have mostly focused on the ability of PET to localize area of hypometabolism overlapping with MCD, but none of them has studied the exact relationship between spatial PET hypometabolism organisation and the spatial organisation of the EZ (beyond the area of the malformation) as defined by stereo-EEG. Using this approach, we found good performance of ${ }^{18}$ F-FDG PET to detect the epileptogenic zone: AUC of 0.82 , sensitivity of 65.8 , specificity of 67.8, PPV of 67.5 and NPV of 87 . The relatively lower performance found here compared to previous studies could be linked to the difference of the methodology we used: namely the accurate comparison of all ROI sampled, rather than the sole correlation with lesion location, primary electro-clinical hypothesis and/or resected zone. Furthermore, three limiting factors exist also in some patients: 1) larger hypometabolic zone than SEEG-defined EZ, 2) limited hypometabolism of certain epileptogenic areas, 3) difficulty in distinguishing between 
epileptogenic and propagation zones. Firstly, our study shows that hypometabolic zone (mean of 20 significantly hypometabolic cortical areas per patient, in comparison with healthy subjects) is often larger than the EZ. Such extension of PET hypometabolism beyond epileptic zones has already been shown in previous studies[22,39,40], in up to $50 \%$ of patients[38]. Moreover, other factors could play a role in PET hypometabolism such as interictal spikes, lifetime number of seizures, and timing of the last seizure role in PET hypometabolism such as interictal spikes, lifetime number of seizures and timing of the last seizure-[41]. These factors were not available in our study, and may explain part of hypometabolism extension. Further studies will be needed to clarify the relationships between PET hypometabolism and these various factors. These factors were not available in our study and then could explain part of the hypometabolism extension.One consequence is the better negative than positive predictive value of PET. Secondly, another aspect of PET misclassification could be due to limited hypometabolism leading to false negative cases. A previous study highlighted that PET hypometabolism could be limited to $10 \%$ of difference compared to the homologous contralateral cortex[12]. Thirdly, our study shows a smaller difference in metabolism between epileptogenic and propagation zones than between epileptogenic and non-involved zones. Consequently, some of the discrepancy between SEEG and PET results are related to misclassification of the propagation zone (50\%).

Despite these limiting factors, our study confirms good performance of PET. We stress important points in our method: 1) we performed PET/SEEG correlation within the ROI sampled by SEEG, 2) the best technique for PET analysis was the use of the peak of hypometabolism and individual normalization per patient. These technical aspects underline the importance in clinical practice of performing PET analysis 1) with a focus on electroclinical hypotheses, 2) with gradual comparison of the degree of hypometabolism at the patient level. 
Taken together, our data point out the difficulty of basing surgical decision on non-invasive data alone in some patients with MCD displaying complex epileptogenic organization. On the one hand, tailoring surgical decision according to significant PET hypometabolism carries the risk of performing unnecessarily large resections. On the other hand, analysis of degree of hypometabolism raises the question of which threshold of hypometabolism should be used to determine EZ. Our study suggests that no absolute threshold could be used and rather an individual integration of electroclinical hypothesis and degree of hypometabolism is most appropriate. Moreover, in such complex cases, SEEG remains mandatory and could be informed by PET data.

\section{Association between PET hypometabolic index and degree of epileptogenicity}

Our paper is the first to demonstrate a graduation in terms of hypometabolism in the context of MCD. In details, we found that the lesional zone was the most hypometabolic zone, followed by the EZ, then the PZ, and finally the NIZ was the less hypometabolic zone. Our paper is the first to demonstrate a graduation in terms of hypometabolism in the context of ACD. We found increasing hypometabolism from non-involved to propagation zones, thento epileptogenic and lesional zones. We used the PET hypometabolic index because of 1) it has good performance in multivariate model for prediction of EZ (see above); 2) it limits the influence of extreme values and improves inter-patient comparison; 3) it favours comparison with the Epileptogenicity Index used in clinical practice (since El is also normalized between 0 and 1). A previous study failed to demonstrate correlation between degree of epileptogenicity and PET metabolism in extra-temporal areas[23]. Our study highlights that, rather than the absolute features of ictal discharge, relative organization of the ictal network correlates best 
with PET metabolism. PET and SEEG data evaluated as normalized indices (ranging from 0-1) per patients gave the best performance. This indeed seems logical given the diversity of spatial organization of epileptogenicity seen in clinical practice from an intracerebral perspective. This is an important methodological point showing the interest of focusing PET analysis on electroclinical hypotheses and of normalizing per patient.

On the other hand, we stress the gradual PET metabolism alteration from lesion to epileptogenic then propagation and finally non-involved zones. This could explain some limitations in PET performance with risk of misclassification. These findings are consistent with the hypothesis of a continuum of epileptogenicity from the most epileptogenic regions to the less epileptogenic regions[33], again challenging the notion of an epileptic focus in favour of more complex epileptogenic network organisation. These results are also consistent with those of our previous studies showing that interictal properties of epileptogenic and propagation zones (defined from ictal recordings) are also similar in terms of changes in structural[32] and functional[29] connectivity.

As previous studies suggested[12], we found small differences in metabolism between FCD type I and II but without statistical significance after multivariate- testing. Moreover, the gradient of metabolism across SEEG-defined zones remains similar across histological type. This last finding suggests that the organization of PET hypometabolism is mainly based on spatial organization of epileptogenicity, independently of histological type-or other clinical characteristics. It suggests a general effect of epilepsy on interictal brain metabolism across diverse $M C D$, and it would be interesting to investigate such general behaviour in other forms of epilepsy. We also found differences in the metabolism of PZ and NIZ according to the visibility of the malformation on MRI, with lower metabolism in MRI-negative cases. It can be due to the more widespread cortical atrophy and connectivity/networks alterations found in 
subtle FCD type I [42-44], which could have been considered as MRI-negative in our study (especially for the patient with the oldest lower field MRI).

\section{Relation with post-surgical prognosis}

Finally, our study demonstrated that larger extension of PET hypometabolism is associated with poorer prognosis for patients with MCD. Such relations between extension of PET hypometabolism and post-surgical seizure outcome has recently emerged in the literature on temporal lobe [45-47] and also neocortical epilepsy [48]. It could be a signature of more widespread cortical alterations beyond areas generating seizure. Such large-scale alteration has been demonstrated in connectivity studies[49-55], and could be responsible for some comorbidities found in patients with focal pharmacoresistant epilepsy, such as cognitive impairment or psychiatric comorbidities. This finding gives additional support suggesting a wider alteration of brain networks beyond the visible MCD in patients with unfavourable postsurgical seizure outcome [25].

In the light of these data, the inclusion of PET data in presurgical outcome estimation seems very useful and, when required, SEEG planning should sampled hypometabolic areas. Further studies on this topic would be of interest in other forms of epilepsy.

\section{Methodological Considerations}

We would like to acknowledge that our study concerned patients with MCDs who required SEEG recordings. This could represent a bias toward a specific subpopulation of patients with MCD with particularly complex epilepsy organization, doubtful/negative MRI or proximity of functional cortex. 
Interestingly, we evaluated PET metabolism with various features: unilateral vs asymmetric metabolism, normalization on cerebellar vs global activity, and T-peak vs patient's index. We performed PET and SEEG registration at the level of the same brain area based on a similar anatomical atlas in order to fit with clinical practice. This approach constitutes an important methodological difference regarding previous studies.

Our multivariate analysis showed that PET performance is independent of other clinical variables. It is consistent with previous literature showing similar performance in FCD type I and II[56].

\section{CONCLUSION:}

${ }^{18}$ F-FDG-PET has good performance to estimate the epileptogenic zone, showing a gradient of hypometabolic index from non-involved to propagation then epileptogenic and lesional zones. Nevertheless, clinicians should be aware that the area of hypometabolism can be larger than the EZ as defined by SEEG quantification. Furthermore, global extension of the hypometabolic zone is negatively associated with post-surgical outcome.

\section{ACKNOWLEDGEMENTS:}

We thank Prof. Figarella-Branger for the reviewing of histological data. We thank Prof. Patrick Chauvel, Prof. Martine Gavaret, Dr. Francesca Bonini, Dr. Lisa Vaugier, Dr. Francesca Pizzo, Dr. Sandrine Aubert, Dr. Geraldine Daquin, Dr. Nathalie Villeneuve and Dr. Anne Lepine (Marseille), for the clinical management of some included patients. We thank Prof. Henry 
Dufour for surgical procedures and Prof. Jean Régis for SEEG procedures, in some of the selected patients.

This work has been carried out within the FHU EPINEXT and DHU-Imaging with the support of the A*MIDEX project (ANR-11-IDEX-0001-02) funded by the "Investissements d'Avenir" French Government program managed by the French National Research Agency (ANR). Part of this work was funded by a joint Agence Nationale de la Recherche (ANR) and Direction Génerale de l'Offre de Santé (DGOS) under grant "VIBRATIONS" ANR-13-PRTS-0011-01

\section{AUTHORS CONTRIBUTIONS}

Stanislas Lagarde, Fabrice Bartolomei and Eric Guedj participated in the Conceptualization and the Methodology of this study.

Stanislas Lagarde, Mohamed Boucekine, Laurent Boyer, Agnes Trebuchon, Romain Carron, Didier Scavarda, Mahtieu Milh, Aileen McGonigal, Fabrice Bartolomei, Eric Guedj participated in the Investigation and the Formal analysis.

Stanislas Lagarde, Mohamed Boucekine, Laurent Boyer, Agnes Trebuchon, Romain Carron, Didier Scavarda, Mahtieu Milh, Aileen McGonigal, Fabrice Bartolomei, Eric Guedj participated in the Writing - review \& editing.

\section{CONFLICT OF INTEREST}

The authors declare that they have no conflict of interest.

\section{ETHICAL APPROVAL:}

All procedures performed in studies involving human participants were in accordance with the ethical standards of the institutional and/or national research committee (French 
Institute of Health (IRB15226)) and with the 1964 Helsinki declaration and its later amendments or comparable ethical standards.

\section{INFORMED CONSENT:}

Informed consent was obtained from all individual participants included in the study.

\section{REFERENCES}

1. Blumcke I, Spreafico R, Haaker G, Coras R, Kobow K, Bien CGCGCG, et al. Histopathological Findings in Brain Tissue Obtained during Epilepsy Surgery. N Engl J Med. 2017;377:1648-56. 2. Cloppenborg T, May TW, Blümcke I, Grewe P, Hopf LJ, Kalbhenn T, et al. Trends in epilepsy surgery: stable surgical numbers despite increasing presurgical volumes. J Neurol Neurosurg Psychiatry. 2016;1-8. 
3. Baud MO, Perneger T, Attila R. European trends in epilepsy surgery. Neurology. 2018;112.

4. Guerrini R, Duchowny M, Jayakar P, Krsek P, Kahane P, Tassi L, et al. Diagnostic methods and treatment options for focal cortical dysplasia. Epilepsia. 2015;56:1669-86.

5. Rowland NC, Englot DJ, Cage TA, Sughrue ME, Barbaro NM, Chang EF. A meta-analysis of predictors of seizure freedom in the surgical management of focal cortical dysplasia. J Neurosurg. 2012;116:1035-41.

6. Faramand AM, Barnes N, Harrison S, Gunny R, Jacques T, Tahir ZM, et al. Seizure and cognitive outcomes after resection of glioneuronal tumors in children. Epilepsia. 2017;1-9. 7. Englot DJ, Berger MS, Barbaro NM, Chang EF. Factors associated with seizure freedom in the surgical resection of glioneuronal tumors. Epilepsia. 2012;53:51-7.

8. Blümcke I, Thom M, Aronica E, Armstrong DD, Vinters H V., Palmini A, et al. The clinicopathologic spectrum of focal cortical dysplasias: A consensus classification proposed by an ad hoc Task Force of the ILAE Diagnostic Methods Commission. Epilepsia. 2011;52:158-74.

9. Giulioni M, Marucci G, Pelliccia V, Gozzo F, Barba C, Didato G, et al. Epilepsy surgery of "low grade epilepsy associated neuroepithelial tumors": A retrospective nationwide Italian study. Epilepsia. 2017;1-10.

10. Louis DN, Perry A, Reifenberger G, von Deimling A, Figarella-Branger D, Cavenee WK, et al. The 2016 World Health Organization Classification of Tumors of the Central Nervous System: a summary. Acta Neuropathol. Springer Berlin Heidelberg; 2016;131:803-20. 11. Isnard J, Taussig D, Bartolomei F, Bourdillon P, Catenoix H, Chassoux F, et al. French guidelines on stereoelectroencephalography (SEEG). Neurophysiol Clin. Elsevier Masson SAS; 2017; 
12. Salamon N, Kung J, Shaw SJ, Koo J, Koh S, Wu JY, et al. FDG-PET/MRI coregistration improves detection of cortical dysplasia in patients with epilepsy. Neurology. 2008;71:1594601.

13. Chassoux F, Landré E, Mellerio C, Turak B, Mann MW, Daumas-Duport C, et al. Type II focal cortical dysplasia: Electroclinical phenotype and surgical outcome related to imaging. Epilepsia. 2012;53:349-58.

14. Desarnaud S, Mellerio C, Semah F, Laurent A, Landre E, Devaux B, et al. 18F-FDG PET in drug-resistant epilepsy due to focal cortical dysplasia type 2: additional value of electroclinical data and coregistration with MRI (European Journal of Nuclear Medicine and Molecular Imaging, (2018), 45, 8, (1449-1460), 10. Eur J Nucl Med Mol Imaging. 2018;45:1465.

15. Rubí S, Setoain X, Donaire A, Bargalló N, Sanmartí F, Carreño M, et al. Validation of FDGPET/MRI coregistration in nonlesional refractory childhood epilepsy. Epilepsia.

2011;52:2216-24.

16. Lee SK, Choe G, Hong KS, Nam HW, Kim JY, Chung CK, et al. Neuroimaging findings of cortical dyslamination with cytomegaly. Epilepsia. 2001;42:850-6.

17. Chugani HT, Shields WD, Shewmon DA, Olson DM, Phelps ME, Peacock WJ. Infantile spasms: I. PET identifies focal cortical dysgenesis in cryptogenic cases for surgical treatment. Ann Neurol. 1990;27:406-13.

18. Kim YK, Lee DS, Lee SK, Chung CK, Chung J-K, Lee MC. (18)F-FDG PET in localization of frontal lobe epilepsy: comparison of visual and SPM analysis. J Nucl Med. 2002;43:1167-74. 19. Kim YH, Kang HC, Kim DS, Kim SH, Shim KW, Kim HDH, et al. Neuroimaging in identifying focal cortical dysplasia and prognostic factors in pediatric and adolescent epilepsy surgery. Epilepsia. 2011;52:722-7. 
20. Casse R, Rowe CCCC, Newton M, Berlangieri SUSU, Scott AMAM. Positron emission tomography and positron emission. Mol Imaging Biol. 2004;6:72-3.

21. Kudr M, Krsek P, Marusic P, Tomasek M, Trnka J, Michalova K, et al. SISCOM and FDG-PET in patients with non-lesional extratemporal epilepsy: Correlation with intracranial EEG, histology, and seizure outcome. Epileptic Disord. 2013;15:3-13.

22. Juhász C, Chugani DC, Muzik O, Watson C, Shah J, Shah A, et al. Is epileptogenic cortex truly hypometabolic on interictal positron emission tomography? Ann Neurol. 2000;48:8896.

23. Lamarche F, Job AS, Deman P, Bhattacharjee M, Hoffmann D, Gallazzini-Crépin C, et al. Correlation of FDG-PET hypometabolism and SEEG epileptogenicity mapping in patients with drug-resistant focal epilepsy. Epilepsia. 2016;57:2045-55.

24. Alkonyi B, Juhász C, Muzik O, Asano E, Saporta A, Shah A, et al. Quantitative brain surface mapping of an electrophysiologic/metabolic mismatch in human neocortical epilepsy.

Epilepsy Res. 2009;87:77-87.

25. Aubert S, Wendling F, Regis J, McGonigal A, Figarella-Branger D, Peragut JC, et al. Local and remote epileptogenicity in focal cortical dysplasias and neurodevelopmental tumours. Brain. 2009;132:3072-86.

26. Lagarde S, Bonini F, McGonigal A, Chauvel P, Gavaret M, Scavarda D, et al. Seizure-onset patterns in focal cortical dysplasia and neurodevelopmental tumors: Relationship with surgical prognosis and neuropathologic subtypes. Epilepsia. 2016;57:1426-35.

27. Jayakar P, Gotman J, Harvey AS, Palmini A, Tassi L, Schomer D, et al. Diagnostic utility of invasive EEG for epilepsy surgery: Indications, modalities, and techniques. Epilepsia. $2016 ; 57: 1735-47$.

28. Engel J. Update on surgical treatment of the epilepsies. Summary of the Second 
International Palm Desert Conference on the Surgical Treatment of the Epilepsies (1992). Neurology. 1993;43:1612-7.

29. Lagarde S, Roehri N, Lambert I, Trebuchon A, McGonigal A, Carron R, et al. Interictal stereotactic-EEG functional connectivity in refractory focal epilepsies. Brain. 2018;1-15. 30. Medina Villalon S, Paz R, Roehri N, Lagarde S, Pizzo F, Colombet B, et al. EpiTools, A software suite for presurgical brain mapping in epilepsy: Intracerebral EEG. J Neurosci Methods. Elsevier B.V.; 2018;303:7-15.

31. Tzourio-Mazoyer N, Landeau B, Papathanassiou D, Crivello F, Etard O, Delcroix N, et al. Automated Anatomical Labeling of Activations in SPM Using a Macroscopic Anatomical Parcellation of the MNI MRI Single-Subject Brain. Neuroimage. 2002;15:273-89.

32. Besson P, Bandt SKK, Proix T, Lagarde S, Jirsa VK, Ranjeva J-PJ-P, et al. Anatomic consistencies across epilepsies: a stereotactic-EEG informed high-resolution structural connectivity study. Brain. 2017;140.

33. Bartolomei F, Chauvel P, Wendling F. Epileptogenicity of brain structures in human temporal lobe epilepsy: A quantified study from intracerebral EEG. Brain. 2008;131:1818-30. 34. Proix T, Bartolomei F, Guye M, Jirsa VK. Individual brain structure and modelling predict seizure propagation. Brain. 2017;140:641-54.

35. Lieberman MD, Cunningham WA. Type I and Type II error concerns in fMRI research: Rebalancing the scale. Soc Cogn Affect Neurosci. 2009;

36. Fluss R, Faraggi D, Reiser B. Estimation of the Youden Index and its associated cutoff point. Biometrical J. 2005;

37. Perkins NJ, Schisterman EF. The Youden index and the optimal cut-point corrected for measurement error. Biometrical J. 2005;

38. Chassoux F, Rodrigo S, Semah F, Beuvon F, Landre E, Devaux B, et al. FDG-PET improves 
surgical outcome in negative MRI Taylor-type focal cortical dysplasias. Neurology.

2010;75:2168-75.

39. Hong SB, Han HJ, Roh SY, Seo DW, Kim SE, Kim MH. Hypometabolism and interictal spikes during positron emission tomography scanning in temporal lobe epilepsy. Eur Neurol. 2002;48:65-70.

40. Juhasz C, Chugani DC, Muzik O, Watson C, Shah J, Shah A, et al. Electroclinical correlates of flumazenil and fluorodeoxyglucose PET abnormalities in lesional epilepsy. Neurology. $2000 ; 55: 825-35$.

41. Tepmongkol S, Srikijvilaikul T, Vasavid P. Factors affecting bilateral temporal lobe hypometabolism on 18F-FDG PET brain scan in unilateral medial temporal lobe epilepsy. Epilepsy Behav. Elsevier Inc.; 2013;29:386-9.

42. Hong SJ, Bernhardt BC, Schrader DS, Bernasconi N, Bernasconi A. Whole-brain MRI phenotyping in dysplasia-related frontal lobe epilepsy. Neurology. 2016;86:643-50.

43. Hong SJ, Lee HM, Gill R, Crane J, Sziklas V, Bernhardt BC, et al. A connectome-based mechanistic model of focal cortical dysplasia. Brain. 2019;142:688-99.

44. Krsek P, Maton B, Korman B, Pacheco-Jacome E, Jayakar P, Dunoyer C, et al. Different features of histopathological subtypes of pediatric focal cortical dysplasia. Ann Neurol. 2008;63:758-69.

45. Chassoux F, Artiges E, Semah F, Laurent A, Landré E, Turak B, et al. ${ }^{18}$ F-FDG-PET patterns of surgical success and failure in mesial temporal lobe epilepsy. Neurology. 2017;88:104553.

46. Cahill V, Sinclair B, Malpas CB, McIntosh AM, Chen Z, Vivash LE, et al. Metabolic patterns and seizure outcomes following anterior temporal lobectomy. Ann Neurol. 2019;1-10.

47. Higo $T$, Sugano $H$, Nakajima $M$, Karagiozov $K$, limura $Y$, Suzuki $M$, et al. The predictive 
value of FDG-PET with 3D-SSP for surgical outcomes in patients with temporal lobe epilepsy. Seizure. BEA Trading Ltd; 2016;41:127-33.

48. Wong CH, Bleasel A, Wen L, Eberl S, Byth K, Fulham M, et al. Relationship between preoperative hypometabolism and surgical outcome in neocortical epilepsy surgery. Epilepsia. 2012;53:1333-40.

49. Besson P, Dinkelacker V, Valabregue R, Thivard L, Leclerc X, Baulac M, et al. Structural connectivity differences in left and right temporal lobe epilepsy. Neuroimage. Elsevier Inc.; 2014;100:135-44.

50. Keller SS, Glenn GR, Weber B, Kreilkamp BAK, Jensen JH, Helpern JA, et al. Preoperative automated fibre quantification predicts postoperative seizure outcome in temporal lobe epilepsy. Brain. 2017;140:68-82.

51. Baker NL, Nesland T, Drane DL, Bonilha L, Breedlove J, Lin JJ, et al. The brain connectome as a personalized biomarker of seizure outcomes after temporal lobectomy The brain connectome as a personalized biomarker of seizure outcomes after temporal lobectomy. Neurology. 2015;1-8.

52. He X, Doucet GE, Pustina D, Sperling MR, Sharan AD, Tracy JI. Presurgical thalamic hubness predicts surgical outcome in temporal lobe epilepsy. Neurology. 2017;88:2285-93. 53. Bonilha L, Nesland T, Martz GU, Joseph JE, Spampinato M V., Edwards JC, et al. Medial temporal lobe epilepsy is associated with neuronal fibre loss and paradoxical increase in structural connectivity of limbic structures. J Neurol Neurosurg Psychiatry. 2012;83:903-9. 54. Bonilha L, Helpern JA, Sainju R, Nesland T, Edwards JC, Glazier SS, et al. Presurgical connectome and postsurgical seizure control in temporal lobe epilepsy. Neurology. 2013;81:1704-10.

55. Bernhardt BC, Hong S, Bernasconi A, Bernasconi N. Imaging structural and functional 
brain networks in temporal lobe epilepsy. Front Hum Neurosci. 2013;7:1-14.

56. Halac G, Delil S, Zafer D, Isler C, Uzan M, Comunoglu N, et al. Compatibility of MRI and FDG-PET findings with histopathological results in patients with focal cortical dysplasia.

Seizure. 2017;45:80-6.

\section{FIGURE LEGENDS}

Figure 1: Receiver Operating Characteristic (ROC) curve of the prediction-models

This figure shows the sensitivity according to the specificity of the statistical model predicting the epileptogenicity (SEEG-based) from PET metabolism data 
Figure 2: Matrix showing the correspondence between model predictions (based on PET) and SEEG epileptogenicity.

The colour code expresses the correspondence: false positive, false negative, no-good predictionerror, area not sampled. ROI= region of interest (from AAL atlas, left and right ROI are fused for graphical representation).

For this figure, we can observe some patients with bad performance of the predicting model (e.g. P7, P16, P18, $\mathrm{P} 36$ with about $50 \%$ of error), and some others with perfect prediction (e.g.

\section{P5, P8, P25, P26, P30, P33).}

\section{Figure 3: Hypometabolic Index according to the considered zone and histology}

Boxplot showing the hypometabolic index (computed from T-Peak Proportional Scaling PET normalized per patient). Panel A exhibited the difference between lesion, epileptogenic (EZ), propagation (PZ) and non-involved zone (NIZ) for all patients. $p$-value are adjusted for multiple comparison. Panel B shows similar gradient of hypometabolic index across all histological types.

Figure 4: Relation between hypometabolism extension and post-surgical seizure outcome. Boxplot showing the percent of significantly hypometabolic areas (including a least one voxel with $p<0.005$, corrected for the cluster volume, on SPM(T) maps) within all the cortical outside SEEG sampling according to the post-surgical seizure outcome. Favourable seizure outcome grouped Engel I and II classes.

Figure 5: Example of relationship between extension of PET hypometabolism and epileptogenicity in SEEG according to the post-surgical seizure outcome.

Panel A shows a zone of hypometabolism limited to areas sampled by the SEEG in a patient which was seizure-free after a post-central cortectomy $(p<0.005, k>119)$, and panel B the 3D representation of SEEG electrodes with the degree of epileptogenicity of each sampled 
region.

Panel C shows a very large zone of hypometabolism, bilateral and exceeding the areas sampled by the SEEG in a patient which was not improve by a premotor cortectomy $(p<0.005$, $k>119$ ), and panel $D$ the 3D representation of SEEG electrodes with the degree of epileptogenicity of each sampled region.

\section{TABLES}

Table 1: Population characteristics

\begin{tabular}{|c|c|}
\hline \multicolumn{2}{|c|}{ Patient's Features } \\
\hline Number of patients & 38 \\
(younger than 18 years old) & (14) \\
\hline
\end{tabular}




\begin{tabular}{|c|c|}
\hline Gender (W/M) & $15(39.5 \%) / 23(60.5 \%)$ \\
\hline Age at epilepsy onset (mean \pm SD) & $7.4 \pm 6.4$ \\
\hline Age at SEEG (mean \pm SD) & $24.8 \pm 13.3$ \\
\hline Epilepsy duration (mean \pm SD) & $17.5 \pm 11.3$ \\
\hline Histology & $\begin{array}{c}\text { FCD I: } 10 \text { (26.3\%) } \\
\text { FCD II: } 21 \text { (55.3\%) } \\
\text { NDT: } 7 \text { (18.4\%) }\end{array}$ \\
\hline Visible MRI lesion & $33(86.8 \%)$ \\
\hline
\end{tabular}

Abbreviations: W: women, M: men, SD: standard-deviation, SEEG: stereo-EEG, FCD: focal cortical dysplasia, NDT: neurodevelopmental tumours

Table 2: Results of the logistic regression analysis testing predictors of epileptogenicitystatistical

* variables included in the multivariate analyses

${ }^{\circ}$ final predicting model included these 2 variablesresults-of variables tested as predictors fort PET metabolism in the model

\begin{tabular}{|l|c|c|c|c|}
\hline Predictors & \multirow{2}{*}{ p-value } & OR & \multicolumn{2}{|c|}{$95 \%$ C.I.for OR } \\
\cline { 4 - 4 } & & Lower & Upper \\
\hline
\end{tabular}

Mis en forme : Police :Gras

Mis en forme : Police :Non Gras

Mis en forme : Police :Non Gras

Mis en forme : Police :Non Gras

Mis en forme : Retrait : Gauche : $0 \mathrm{~cm}$, Première ligne : 0 $\mathrm{cm}$, Interligne : 1,5 ligne

Mis en forme : Police :Gras

Tableau mis en forme 


\begin{tabular}{|c|c|c|c|c|c|}
\hline \multirow{7}{*}{$\begin{array}{c}\text { Clinical } \\
\text { Variable }\end{array}$} & Age of onset & 0.328 & 0.979 & 0.938 & 1.021 \\
\hline & Epilepsy duration & 0.581 & 1.006 & 0.984 & 1.029 \\
\hline & Age at SEEG & 0.965 & 1.000 & 0.981 & 1.020 \\
\hline & MRI-positive?positive? * & 0.083 & 2.047 & 0.912 & 4.598 \\
\hline & Gender* & 0.088 & 0.629 & 0.369 & 1.072 \\
\hline & Localization of MCD* & 0.034 & 0.198 & 0.044 & 0.885 \\
\hline & Histology* & 0.008 & 0.455 & 0.254 & 0.817 \\
\hline \multirow{9}{*}{ PET variables } & $\begin{array}{c}\text { Average metabolic ROI } \\
\text { value after proportional } \\
\text { scaling* }\end{array}$ & $<0.001$ & 0.948 & 0.926 & 0.971 \\
\hline & $\begin{array}{c}\text { Average metabolic ROI } \\
\text { value after cerebellar } \\
\text { scaling* }\end{array}$ & $<0.001$ & 0.031 & 0.007 & 0.130 \\
\hline & Metabolic asymmetry* & $<0.001$ & 0.850 & 0.784 & 0.921 \\
\hline & $\begin{array}{l}\text { Average metabolic ROI } \\
\text { value expressed as Z-score } \\
\text { in comparison to controls } \\
\text { after proportional scaling* }\end{array}$ & $<0.001$ & 0.680 & 0.574 & 0.805 \\
\hline & $\begin{array}{l}\text { Average metabolic ROI } \\
\text { value expressed as Z-score } \\
\text { in comparison to controls } \\
\text { after cerebellar scaling* }\end{array}$ & $<0.001$ & 0.684 & 0.587 & 0.797 \\
\hline & $\begin{array}{c}\text { Voxel T-peak after } \\
\text { proportional scaling* }\end{array}$ & $<0.001$ & 1.750 & 1.457 & 2.101 \\
\hline & $\begin{array}{l}\text { Metabolic index after } \\
\text { proportional scaling* }\end{array}$ & $<0.001$ & 29.046 & 9.174 & 91.960 \\
\hline & $\begin{array}{l}\text { Voxel T-peak after } \\
\text { cerebellar scaling* }\end{array}$ & $<0.001$ & 1.609 & 1.383 & 1.871 \\
\hline & $\begin{array}{c}\text { Metabolic index after } \\
\text { cerebellar scaling* }\end{array}$ & 0.099 & 0.491 & 0.211 & 1.143 \\
\hline \multicolumn{6}{|c|}{ Significant variables after Multivariate Analysis_ ${ }_{-}^{\circ}$} \\
\hline \multirow{2}{*}{ PET variables } & $\begin{array}{c}\text { Metabolic index after } \\
\text { proportional scaling }\end{array}$ & 0.017 & 4.89 & 1.325 & 18.047 \\
\hline & $\begin{array}{l}\text { Voxel T-peak after } \\
\text { cerebellar scaling }\end{array}$ & $<0.001$ & 1.473 & 1.248 & 1.738 \\
\hline
\end{tabular}

